\title{
The MAP Kinase Kinase Gene AbSte 7 Regulates Multiple Aspects of Alternaria brassicicola Pathogenesis
}

\author{
Kai Lu', Min Zhang', Ran Yang ${ }^{1}$, Min Zhang ${ }^{1}$, Qinjun Guo ${ }^{1}$, Kwang-Hyun Baek ${ }^{2 *}$, and Houjuan Xu ${ }^{1 *}$ \\ ${ }^{1}$ Department of Plant Protection, Shandong Agricultural University, Tai'an 271018, China \\ ${ }^{2}$ Department of Biotechnology, Yeungnam University, Gyeongsan, Gyeongbuk 38541, Korea
}

(Received on July 5, 2018; Revised on November 15, 2018; Accepted on December 6, 2018)

Mitogen-activated protein kinase (MAPK) cascades in fungi are ubiquitously conserved signaling pathways that regulate stress responses, vegetative growth, pathogenicity, and many other developmental processes. Previously, we reported that the AbSte 7 gene, which encodes a mitogen-activated protein kinase kinase (MAPKK) in Alternaria brassicicola, plays a central role in pathogenicity against host cabbage plants. In this research, we further characterized the role of $\mathrm{AbSte} 7$ in the pathogenicity of this fungus using $\triangle A B S t e 7$ mutants. Disruption of the $\mathrm{AbSte} 7$ gene of $A$. brassicicola reduced accumulation of metabolites toxic to the host plant in liquid culture media. The $\triangle A b S t e 7$ mutants could not efficiently detoxify cruciferous phytoalexin brassinin, possibly due to reduced expression of the brassinin hydrolase gene involved in detoxifying brassinin. Disruption of the $\mathrm{AbSte} 7$ gene also severely impaired fungal detoxification of reactive oxygen species. $\mathrm{AbSte} 7$ gene disruption reduced the enzymatic activity of cell walldegrading enzymes, including cellulase, $\beta$-glucosidase, pectin methylesterase, polymethyl-galacturonase, and polygalacturonic acid transeliminase, during host plant infection. Altogether, the data strongly suggest the MAPKK gene $\mathrm{AbSte} 7$ plays a pivotal role in $\mathrm{A}$. brassici-

\footnotetext{
*Co-corresponding authors.

$\mathrm{H} . \mathrm{Xu}$

Phone) +86-538-824-1575, FAX) +86-538-824-1324

E-mail)xhjuan@sdau.edu.cn

K.-H. Baek

Phone) +82-53-810-3029, FAX) +82-53-810-4769

E-mail)khbaek@ynu.ac.kr

(c) This is an Open Access article distributed under the terms of the Creative Commons Attribution Non-Commercial License (http:/ creativecommons.org/licenses/by-nc/4.0) which permits unrestricted noncommercial use, distribution, and reproduction in any medium, provided the original work is properly cited.
}

Articles can be freely viewed online at www.ppjonline.org. cola during host infection by regulating multiple steps, and thus increasing pathogenicity and inhibiting host defenses.

Keywords : AbSte7, Alternaria brassicicola, mitogenactivated protein kinase kinase (MAPKK), pathogenicity

Handling Editor: Sohn, Kee Hoon

Mitogen-activated protein kinase (MAPK) cascades are ubiquitous signaling modules in fungi that regulate cellular responses to environmental changes (Pearson et al., 2001). In the budding yeast Saccharomyces cerevisiae, five MAPK signal transduction pathways have been identified and extensively studied. These pathways regulate mating, filamentous growth, high osmolarity responses, maintenance of cell wall integrity, and ascospore formation (Gustin et al., 1998; Widmann et al., 1999). Unlike $S$. cerevisiae and other fungi in Saccharomycetales, most filamentous fungi have only three major MAPK cascades, which are homologous to the yeast Fus3/Kss1, Slt2, and Hog1 pathways (Hamel et al., 2012; Román et al., 2007; Zhao et al., 2007).

Among these three MAPK pathways, the yeast Fus3/ Kss1 homolog pathway has been the most extensively studied (Zhao et al., 2007). Many Fus3/Kss1 homologs characterized in phytopathogenic fungi control a wide variety of developmental processes but have a remarkably conserved role in regulating fungal pathogenesis $(\mathrm{Xu}, 2000$; Zhao et al., 2007). Despite the crucial roles these factors play in fungal pathogenesis, aspects of infection regulated by Fus3/ Kss1 homologs in phytopathogenic fungi are as diverse as the fungi themselves. For example, in Magnaporthe grisea (Xu et al., 1996), Pyrenophora teres (Ruiz-Roldan et al., 2001), Bipolaris oryzae (Moriwaki et al., 2007), Fusarium graminearum (Jenczmionka et al., 2003), and Mycosphae- 
rella graminicola (Cousin et al., 2006), Fus3/Kss1 homologs mutants are completely nonpathogenic on intact host plants, while in Cochliobolus heterostrophus (Lev et al., 1999 ) and $F$. graminearum (Hou et al., 2002), these mutants only have partial inhibition of pathogenicity. Fus3/ Kss1 homolog P. teres (Ruiz-Roldan et al., 2001) and $M$. graminicola mutants (Cousin et al., 2006) are also apathogenic on wounded host leaves. However, mutants of these homologs in A. alternate induce necrotic lesions indistinguishable from those produced by wild-type strains (Lin et al., 2010). These results suggest the Fus3/Kss1 homologs are involved in different infection steps in different fungi.

Alternaria brassicicola (Schwein.) Wiltshire is the causative agent of black spot disease in cruciferous vegetables. Although this disease has significantly reduced vegetable quantity and quality in many countries and become an important factor limiting cultivated Brassica species (Chen et al., 2005; Rop et al., 2009), the mechanisms of A. brassicicola pathogenesis are still not fully understood (Cho, 2015).

Recently, AbSte7 (NCBI accession no. MH559277), which is the $A$. brassicicola equivalent to a MAPKK gene in the yeast Fus 3 pathway, was cloned and functionally characterized in our laboratory. AbSte7 knockout mutants completely lost their ability to infect intact cabbage leaves (Xu et al., 2016), again demonstrating the conserved role of the Fus 3 pathway regulating fungal pathogenesis. In this study, we analyzed the regulation of infection by AbSte7 and increased our understanding of the mechanisms of pathogenesis of A. brassicicola.

\section{Materials and Methods}

Strains, growth conditions, and DNA manipulation. The fungal species $A$. brassicicola abtal4 was originally isolated from infected cabbage leaves (Brassica oleracea) in the providence of Shandong, China and was used as the wild-type (WT) strain in this study. Two $\triangle A b S t e 7$ mutant (M1 and M2) and the $\triangle A b S t e 7$ complement (C1) strains were generated in our lab as previously described (Xu et al., 2016). All strains were cultured on potato dextrose agar (PDA) at $25^{\circ} \mathrm{C}$ for growth and extraction of genomic DNA and RNA. All standard molecular techniques were performed according to the protocols described by Sambrock and Russell (2001).

Preparation of crude broth extract (CBE) and phytotoxicity assay. All strains were incubated in glucose yeast extract agar ( $1 \%$ glucose, $0.5 \%$ yeast extract, and $1 \%$ agar) media at $25^{\circ} \mathrm{C}$ for 5 days, then 50 agar plugs $5 \mathrm{~mm}$ in di- ameter were cut from these plates and transferred to $200 \mathrm{ml}$ of $20 \%$ V8 juice broth in $500 \mathrm{ml}$ Erlenmeyer flasks. After being cultivated for 14 days at $25^{\circ} \mathrm{C}$ in the dark, the cultures were filtered using Whatman no. 1 filter paper (Whatman Co., England) to separate the broth from the mycelia. Crude broth extraction and phytotoxicity assays were conducted according to MacKinnon et al. (1999). The filtered broth was extracted with ethyl acetate and dried over $\mathrm{NaSO}_{4}$ to acquire the CBE. Phytotoxicity assays were conducted with a $5 \%$ methanol solution of CBE on detached cabbage leaves.

Brassinin detoxification and $B H A b$ gene expression in WT and $\triangle A$ AbSte 7 mutants. A. brassicicola WT strain abta 14 and $\triangle A b S t e 7$ mutants were grown on PDA plates at $25^{\circ} \mathrm{C}$ for 4 days. Brassinin detoxification by the strains was determined on PDA media supplemented with 0.1 and 0.2 $\mathrm{mM}$ brassinin. For the control, DMSO $(1 \% \mathrm{v} / \mathrm{v}$ final concentration) was added instead of brassinin. Mycelial plugs (5 $\mathrm{mm}$ in diameter) from 4-day-old PDA plates of the strains were placed on the center of each plate supplemented with brassinin or DMSO. All the plates were incubated at $25^{\circ} \mathrm{C}$ and the colony diameters were measured at 6 days post-inoculation (dpi). All experiments were carried out in triplicate at least three times.

Expression of the brassinin hydrolase gene (BHAb, NCBI accession no. KC814178.1) was examined in the $\triangle A b S t e 7$ mutants and WT strain of $A$. brassicicola during plant infection using quantitative RT-PCR (qRT-PCR). The primer pair $B H A b-f$ and $-r$ (5'-CATCATGCACTGCTCGAACG-3'; 5'-GTGTTGCACGCTTTGGGTAG-3') were designed using the Primer Premier 6.0 program (PREMIER Biosoft International, Palo Alto, CA, USA). The primer pair for the housekeeping gene Actin (NCBI accession no. JQ671669) were also prepared as Actin- $f$ and $-r$ (5'-ATTGAGCACGGTGTCGTT-3'; 5'-TGGTGGAGAAGGTGTAACCG-3'). For RNA extraction, A. brassicicola WT and $\triangle A b S t e 7$ mutant strain mycelia were harvested from PDA plates cultured for 5 days at $25^{\circ} \mathrm{C}$. Cabbage leaves were inoculated with the WT or mutant strain as described by $\mathrm{Xu}$ et al. (2016), then collected at 12, 24, 48, and $72 \mathrm{~h}$ after inoculation. Total RNA was extracted using TRIzol reagent (Invitrogen, Carlsbad, CA, USA) according to the manufacturer's instructions. Reverse transcription was carried out with the PrimeScript ${ }^{\mathrm{TM}} \mathrm{RT}$ reagent kit with gDNA Eraser (Takara, Japan). The qRT-PCR reactions were performed with SYBR ${ }^{\circledR}$ Premix Ex Taq ${ }^{\mathrm{TM}}$ II (Takara, Japan).

$\mathrm{H}_{2} \mathrm{O}_{2}$ detoxification by $A$. brassicicola $\triangle$ AbSte $7 . \mathrm{H}_{2} \mathrm{O}_{2}$ detoxification was determined by measuring the decrease 
in absorbance at $240 \mathrm{~nm}$ with a UV-vis spectrophotometer (TU-1950, Persee, China) (Chen et al., 2012). Five mycelial plugs ( $5 \mathrm{~mm}$ in diameter) of the A. brassicicola WT and $\triangle A b S t e 7$ mutants strains were picked from the periphery of mycelia on the PDA plates, transferred to 50-ml flasks containing $10 \mathrm{ml}$ water and $30 \mathrm{mM} \mathrm{H}_{2} \mathrm{O}_{2}$, and incubated at $25^{\circ} \mathrm{C}$. For the control, a PDA agar plug lacking fungal hyphae was used to inoculate the flasks. The amount of $\mathrm{H}_{2} \mathrm{O}_{2}$ in the solution was monitored spectrophotometrically over time.

Enzymatic assays of cell wall-degrading enzymes. Assays evaluating colony growth in major carbon sources were conducted for $\triangle A b S t e 7$ mutants according to Cho et al. (2015). Mycelial growth of $A$. brassicicola WT and $\triangle A b S t e 7$ mutant strains was assessed in minimal medium supplemented with $1 \%$ citrus pectin (cat\# P9135-100g, Sigma-Aldrich, St. Louis, MO, USA), 1\% xylan (cat\# X823251, Shanghai Macklin Biochemical Co., China), or $1 \%$ cellulose (cat\# C804601, Shanghai Macklin Biochemical Co., China).

A series of cell wall-degrading enzymes (CWDEs), including polygalacturonase $(\mathrm{PG})$, cellulase $(\mathrm{Cx})$, $\beta$-glucosidase $(\beta-\mathrm{G})$, polygalacturonic acid trans-eliminase (PGTE), pectin methyl-trans-eliminase (PMTE), and pectin methylesterase (PME), was detected in hosts infected with the WT and $\triangle A b S t e 7 \mathrm{M} 1$ mutant strains. At 12, 24, 48, and $72 \mathrm{hpi}$, the infected leaves were harvested for crude enzyme extraction and CWDEs were measured according to Yang et al. (2012). All treatments were replicated three times. The data was analyzed using SPSS 22.0 software (SPSS Inc., Chicago, USA). Statistically significant differences between strains were determined by Student's $t$-test $(P<0.05)$.

\section{Results}

CBE of $\triangle A b S t e 7$ has reduced phytotoxicity. $\triangle A b S t e 7$ mutants could not produce fully developed conidia but the secreted toxins of $A$. brassicicola had not been evaluated for the phytotoxicity, therefore, we examined the phytotoxicity of the CBE by inoculating it to the detached cabbage leaves. As shown in Fig. 1A, 5\% methanol control (MA) caused needle lesion only at the site of inoculation, suggesting that the methanol solvent had no effect on the lesion development. Typical chlorotic lesions appeared on leaves inoculated with CBEs from the $A$. brassicicola WT and the complementation strain, which expanded along the vascular system and reached diameters of 10.2 and $9.6 \mathrm{~mm}$ at $6 \mathrm{dpi}$, respectively (Fig. 1B). Inoculation of leaves with

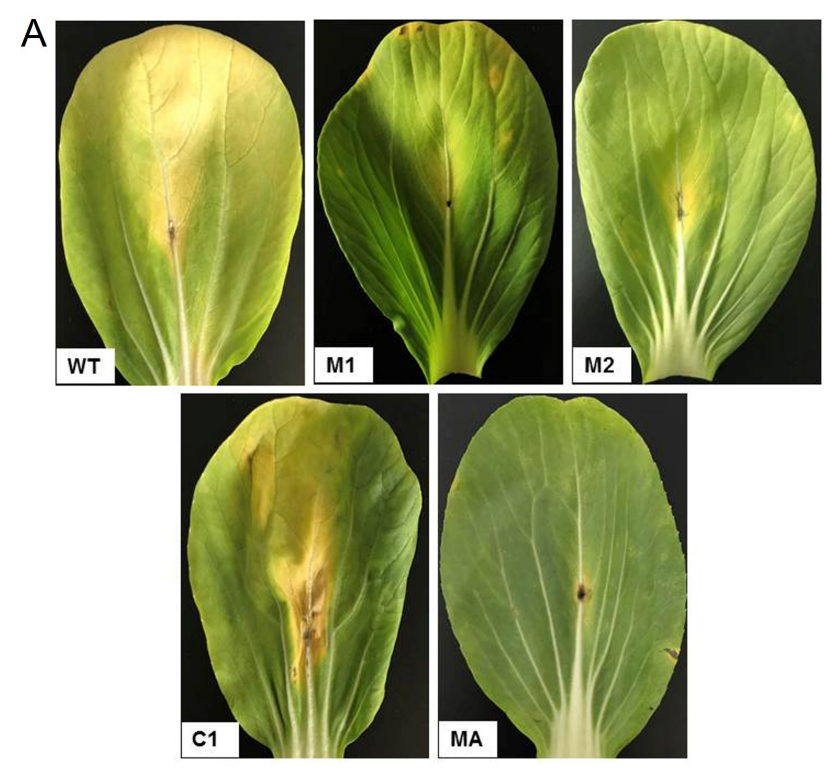

B

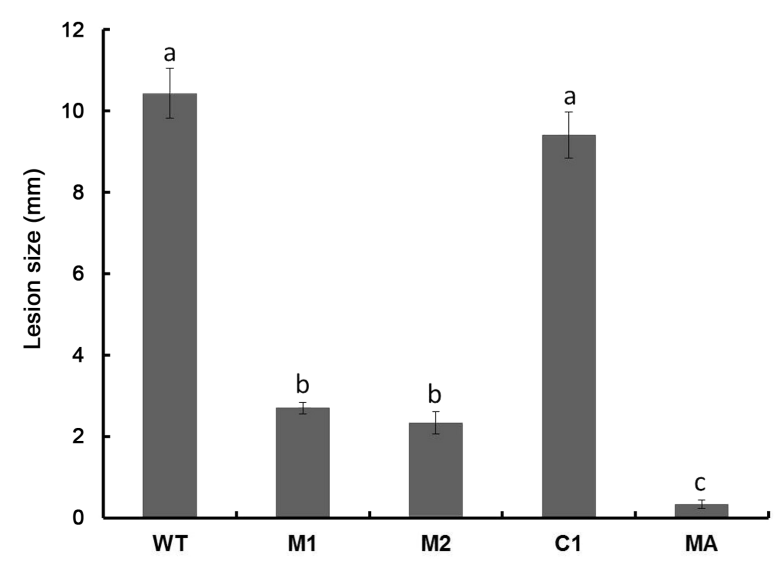

Fig. 1. Phytotoxicity assays of crude broth extracts from different strains. (A) Crude broth extracts from different Alternaria brassicicola strains were inoculated on detached cabbage leaves. WT: wild-type of $A$. brassicicola; $\mathrm{M} 1$ and M2: AbSte 7 disruption mutants; $\mathrm{C} 1: \triangle \mathrm{AbSte} 7$ complementation strain; MA: $5 \%$ methanol control. (B) Lesion size following infection with different $A$. brassicicola strains was recorded at 5 days post-inoculation. Each column contains the mean \pm standard error of three independent experiments with three replicates. The different letters in the column indicate significant differences as determined by Student's ttest $(P<0.05)$.

CBEs from the M1 and M2 strains also resulted in chlorosis at $3 \mathrm{dpi}$; however, the lesions expanded slowly, with diameters of 2.6 and $2.3 \mathrm{~mm}$ at $6 \mathrm{dpi}$, respectively (Fig. 1B).

$\triangle A b S t e 7$ has a reduced ability to detoxify brassinin. Brassinin is an important phytoalexin in Brassica species and plays a significant role in the defense. To determine the deficient steps in the pathogenicity of $\triangle A b S t e 7$, we 
Table 1. Effects of brassinin on the colony growth of $\triangle A b S t e 7$ mutants and wild-type strains of Alternaria brassicicola on potato dextrose agar plates

\begin{tabular}{|c|c|c|c|c|c|c|}
\hline \multirow[b]{2}{*}{ Strains } & \multirow[b]{2}{*}{ PDA } & \multirow{2}{*}{$\begin{array}{c}\mathrm{PDA}+\mathrm{DMSO} \\
(0.05 \%)\end{array}$} & \multicolumn{2}{|c|}{$\mathrm{PDA}+\operatorname{Brassinin}(0.1 \mathrm{mM})$} & \multicolumn{2}{|c|}{$\mathrm{PDA}+\operatorname{Brassinin}(0.2 \mathrm{mM})$} \\
\hline & & & $\begin{array}{l}\text { Colony diameter } \\
\text { (mm) }\end{array}$ & $\begin{array}{c}\text { Growth inhibition } \\
\text { rates }(\%)\end{array}$ & $\begin{array}{l}\text { Colony diameter } \\
\text { (mm) }\end{array}$ & $\begin{array}{c}\text { Growth inhibition } \\
\text { rates }(\%)\end{array}$ \\
\hline WT & $88 \pm 3.2$ & $86 \pm 1.4$ & $86 \pm 4.3$ & - & $84 \pm 3.8$ & - \\
\hline M1 & $68 \pm 1.5$ & $63 \pm 3.1$ & $52 \pm 1.8$ & $23 \%$ & $33 \pm 1.2$ & $51 \%$ \\
\hline M2 & $64 \pm 2.7$ & $66 \pm 1.9$ & $49 \pm 2.5$ & $22 \%$ & $30 \pm 2.2$ & $53 \%$ \\
\hline $\mathrm{C} 1$ & $85 \pm 3.8$ & $90 \pm 3.5$ & $86 \pm 2.2$ & - & $85 \pm 2.4$ & - \\
\hline
\end{tabular}

WT: wild-type, $\mathrm{M} 1$ and M2: two $\triangle A b S t e 7$ mutants, $\mathrm{C} 1: \triangle A b S t e 7$ Complemented mutant.

Numbers represent mean \pm standard error of three replicates.

examined the ability of $\triangle A b S t e 7$ to detoxify brassinin by comparing the colony growth rate on PDA and PDA supplemented with DMSO or brassinin. As shown in Table 1, DMSO used as the solvent for brassinin, had no effect on mycelial growth of $A$. brassicicola WT and $\triangle A b S t e 7$ mutant strains as their colony diameters were similar on PDA with or without DMSO.

However, the abilities of the A. brassicicola WT and $\triangle A b S t e 7$ mutant strains to detoxify brassinin were significantly different. The WT strain grew normally on PDA containing 0.1 or $0.2 \mathrm{mM}$ brassinin with the colony diameter indistinguishable from that of WT strain grown on plain PDA. By contrast, the growth rates of the $\triangle A b S t e 7 \mathrm{M} 1$ and M2 mutants were remarkably reduced in the presence of

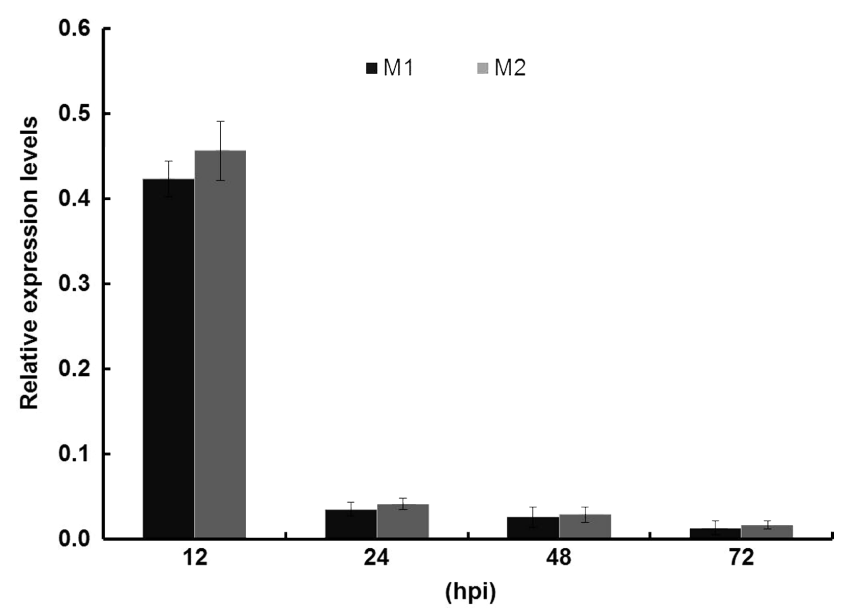

Fig. 2. Relative expression levels of the $B H A b$ gene in AbSte7 disruption mutants M1 and M2 at different time points during infection. The expression levels of $B H A b$ in WT, M1, and M2 were compared to the expression levels of the gene encoding actin for normalization. Relative expression levels were calculated by dividing the expression levels of M1 or M2 by those of WT. The expression levels were calculated using the comparative $\Delta \Delta \mathrm{Ct}$ method. The data are displayed as mean \pm standard error for three independent experiments. brassinin. When grown on 0.1 and $0.2 \mathrm{mM}$ brassinin, the reductions in the colony diameters of M1 were 23 and 51\% and M2 were 22 and 53\%, respectively (Table 1).

We also examined $B H A b$ gene expression level in mutants M1 and M2 during host-pathogen interactions. As shown in Fig. 2, BHAb was expressed during all stages of infection examined, but the expression levels in the $\triangle A b S t e 7$ mutant and WT strains were quite different. Expression in the $\triangle A b S t e 7 \mathrm{M} 1$ and M2 mutants was highest at $12 \mathrm{~h}$ post-inoculation (hpi), but was only approximately 0.4 -fold that of WT. As the infection increased the expression level in the $\triangle A b S t e 7$ mutants decreased dramatically, where expression was only 0.012 - and 0.016 -folds, respectively, that of WT at 72 hpi (Fig. 2).

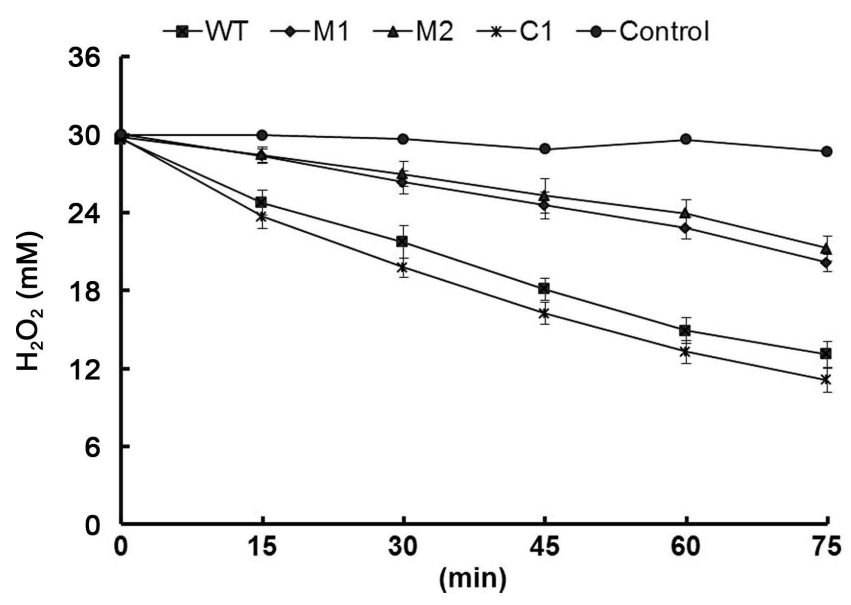

Fig. 3. Spectrophotometric analysis of $\mathrm{H}_{2} \mathrm{O}_{2}$ detoxification by $A$. brassicicola WT and $\triangle A b S t e 7$ mutants. The absorbance at 240 nm was measured for a $30 \mathrm{mM} \mathrm{H}_{2} \mathrm{O}_{2}$ solution following the addition of mycelia plugs of $A$. brassicicola WT or $\triangle A b S t e 7$ mutants M1 and M2 or agar plugs lacking mycelia (control) and incubating for $75 \mathrm{~min}$. The $\triangle A b S t e 7$ mutants had a severely impaired ability to detoxify $\mathrm{H}_{2} \mathrm{O}_{2}$. The data are presented as mean \pm standard error for three independent experiments. 
Disruption of the MAPKK AbSte7 severely impairs detoxification of $\mathrm{H}_{2} \mathrm{O}_{2} \cdot \mathrm{H}_{2} \mathrm{O}_{2}$ detoxification of different strains over time was evaluated spectrophotometrically at an absorbance of $240 \mathrm{~nm}$, the AbSte7 disruption mutants $\mathrm{M} 1$ and $\mathrm{M} 2$ were found to be unable to detoxify $\mathrm{H}_{2} \mathrm{O}_{2}$ effectively. WT degraded $58.82 \%$ of the $\mathrm{H}_{2} \mathrm{O}_{2}$ after an incubation of 75 minutes, while M1 and M2 degraded 32.92 and $28.82 \%$ of the $\mathrm{H}_{2} \mathrm{O}_{2}$, respectively (Fig. 3), indicating that ROS detoxification was severely impaired in the
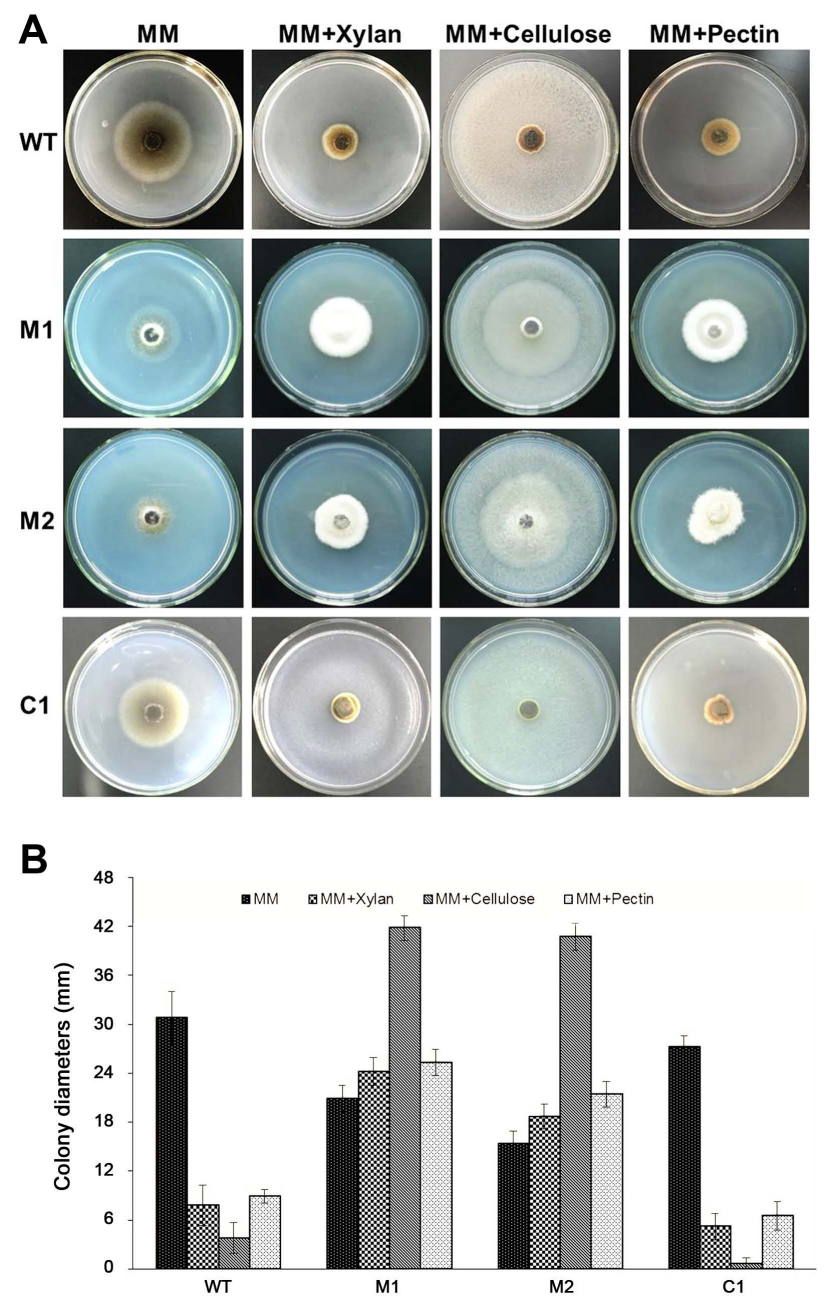

Fig. 4. (A) Carbohydrate utilization by A. brassicicola WT and $\triangle A b S t e 7$ mutant strains. The $\triangle A b S t e 7$ mutant M1 and M2 and WT strains were grown on minimal media supplemented with the carbon sources xylan, cellulose, and pectin at $25^{\circ} \mathrm{C}$ for $7 \mathrm{~d}$. (B) The carbohydrate utilization and hydrolytic enzyme production in $\triangle A b S t e 7$ and WT strains were determined by the growth rates in minimal medium supplemented with diverse carbon sources. The growth rates (D) were calculated as: D = D1-D2. D1 and D2 were the colony diameter measured at 7 days after incubation and the diameter of the agar block for inoculation $(8 \mathrm{~mm})$, respectively. Each column represents the mean $\pm \mathrm{SE}$ of the three independent experiments with three replicates.

\section{$\triangle A b S t e 7$ mutants.}

AbSte 7 is involved in regulation of CWDE activity. The growth rates of the $\triangle A b S t e 7$ mutant and WT strains were measured in minimal medium supplemented with diverse carbon sources to explore carbohydrate utilization and hydrolytic enzyme production. As shown in Fig. 4A and $\mathrm{B}$, the addition of one of three carbohydrates, especially cellulose, to minimal media reduced the growth rates of the WT strain. The $\triangle A b S t e 7$ mutants M1 and M2 grew more quickly on the minimum media (MM) supplemented with all three carbohydrates. Especially the colony diameters of M1 and M2 on MM plus with cellulose were 41.8 and $40.7 \mathrm{~mm}$, respectively, while those of WT were only 4.3 $\mathrm{mm}$ (Fig. 4B). Furthermore, the aerial hyphae were more flourishing in minimal medium when supplemented with xylan and pectin. The growth rates of the complemented strain $\mathrm{C} 1$ in MM plates supplemented with all three carbohydrates were similar with that of WT strain. These results suggested the activities of several hydrolytic enzymes were negatively regulated by AbSte7 during saprophytic growth under nutrient-poor conditions.

We then measured CWDE activity in the WT and $\triangle A b S t e 7$ mutants during host plant infection. As shown in Figure 5A, Cx activity increased from 12 to 24 hpi and then decreased in the WT strain. Cx activity in the M1 and M2 mutants declined progressively, but slightly, from 12 to 72 hpi and was significantly lower than that of WT during the entirety of the assay period except at 12 hpi (Fig. 5A). The $\triangle A b S t e 7$ mutants had lower $\beta-G$ activity than WT for the entire assay period (Fig. 5B). The activity of $\beta-G$ increased during early infection stages in WT by 48 hpi and in M1 by 24 hpi then declined rapidly.

PG activity in the WT and two $\triangle A b S t e 7$ mutants gradually increased but did not significantly differ between the strains (Fig. 5C). PME activity in the WT and $\triangle A b S t e 7$ mutants increased over 72 hpi with significantly higher levels of activity in WT than the mutants (Fig. 5D). The PGTE activity of the WT strain increased dramatically from 12 to 24 hpi and the growth rate was steady, but low, from 24 to 72 hpi. For the $\triangle A b S t e 7$ mutants M1 and M2, PGTE activity peaked at $48 \mathrm{hpi}$, then declined slightly to 72 hpi (Fig. 5E). PMTE activity differed between the WT and $\triangle A b S t e 7$ mutants. It increased constantly from 12 to $72 \mathrm{hpi}$ in WT. However, in the $\triangle A b S t e 7$ mutants, PMTE activity increased from 12 to $24 \mathrm{hpi}$, but then remained approximately the same from 24 to $72 \mathrm{hpi}$ (Fig. 5F). Both the PGTE and PMTE activities were higher in WT than the $\triangle A b S t e 7$ mutants, at later infection stages with no differences observed at $12 \mathrm{hpi}$. 

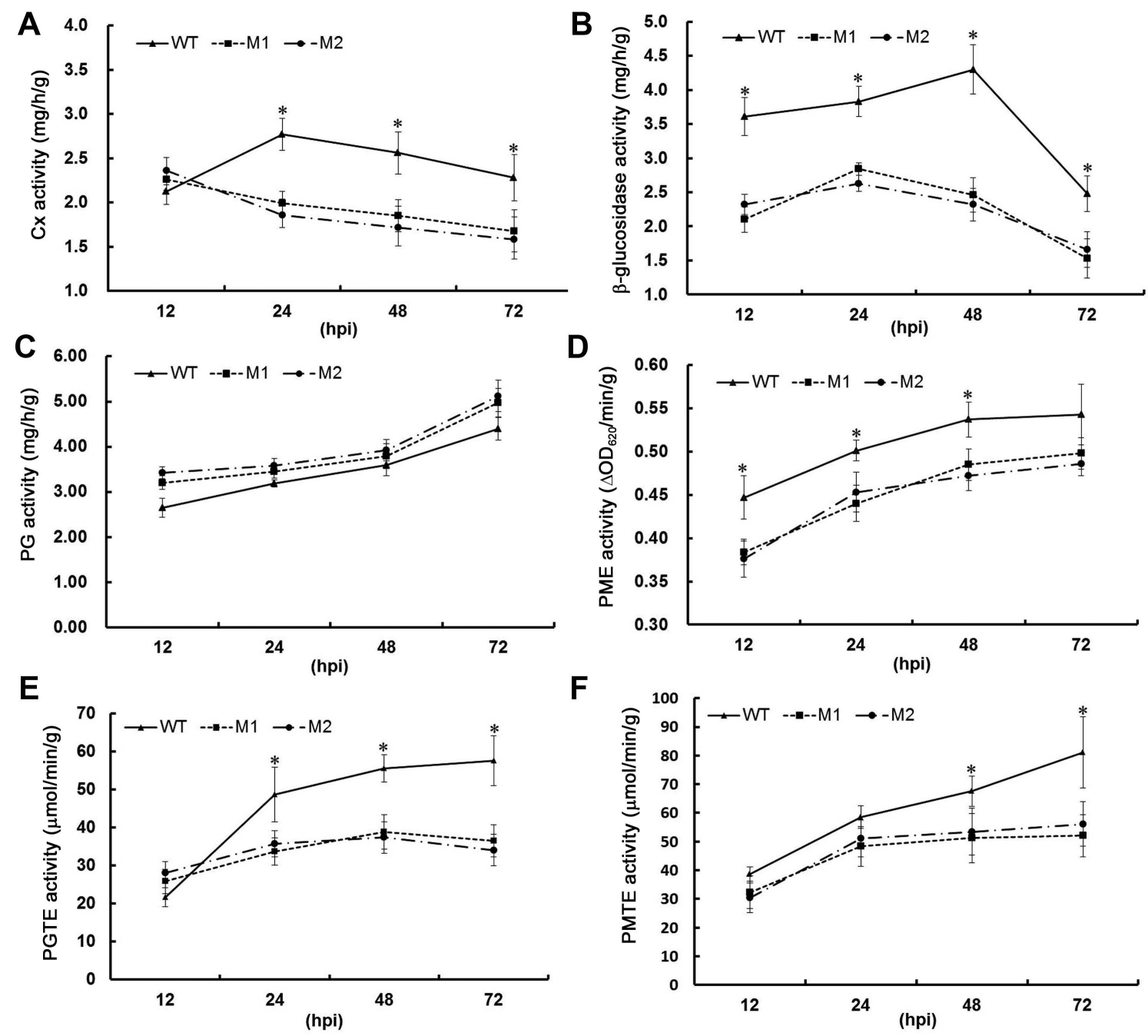

$\mathbf{F}$

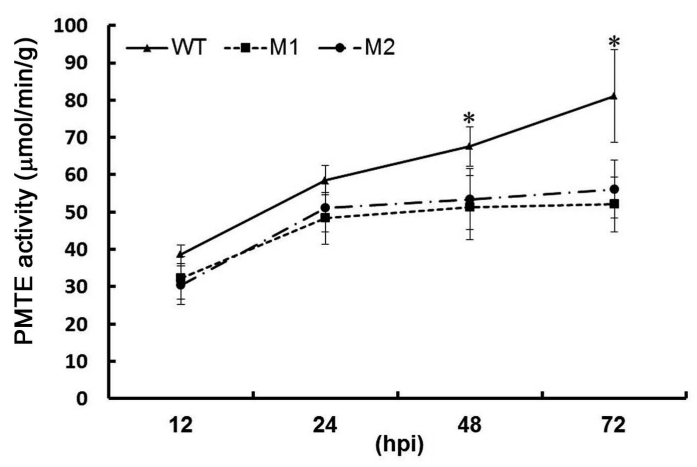

Fig. 5. Enzymatic activity of cellulase (Cx, A), $\beta$-glucosidase (B), polygalacturonase (PG, C), pectin methylesterase (PME, D), polygalacturonic acid trans-eliminase (PGTE, E) and pectin methyl-trans-eliminase (PMTE, F) in infected cabbage leaves after inoculation with the $A$. brassicicola WT or $\triangle A b S t e 7 \mathrm{M} 1$ mutant strains. Bars represent standard errors of the mean. Means with an asterisk $(*)$ indicate a significant difference at $P \leq 0.05$.

\section{Discussion}

The phytopathogenic Alternaria species usually produce host- and/or nonhost-specific toxins to damage plant tissues and facilitate colonization (Nishimura and Kohmoto, 1983; Thomma, 2003). However, the roles of A. brassicicola toxin (AB-toxin) on pathogenesis have not been completely characterized. AB-toxin, a host-specific protein toxin of A. brassicicola, is produced by germinating spores but not on culture media (Otani et al., 1998). AB-toxin is induced by recognition of host-derived oligosaccharides, but the encoding gene remains unknown and its specific function in pathogenesis remains unclear (Oka et al., 2005). Isolation of phytotoxic metabolites showed brassicicolin $\mathrm{A}$ is the most selective phytotoxic metabolite produced in the liquid cultures of A. brassicicola (Pedras et al., 2009; Pedras and Park, 2015). Comparison of phytotoxicity of CBEs from $\triangle A b S t e 7$ mutant and WT strains indicates that disruption of the AbSte7 gene reduced phytotoxic metabolite accumulation in culture media (Fig. 1). Because AB-toxin could not be produced in culture media, it was not responsible for the reduced phytotoxicity of the CBEs in the $\triangle A b S t e 7$ culture media. Instead, these results suggest that disruption of Abste7 reduces levels of toxic metabolites, such as the phytotoxic metabolite brassicicolin A, in culture media. This reduction may be responsible, at least in part, for the reduced virulence of the $\mathrm{M} 1$ and $\mathrm{M} 2$ mutants.

Brassinin is an important phytoalexin produced by crucifers in response to the fungal attack and other forms of 
stress (Pedras et al., 2010, 2012). Its antifungal activity is partly a result of its dithiocarbamate group, which has extensively been used in pesticides and fungicides (Pedras et al., 2012; Szolar, 2007). To overcome the phytoalexin, A. brassicicola produces brassinin hydrolase, which detoxifies brassinin into the intermediate metabolites $\mathrm{N}^{\prime}$ indolylmethanamine and $\mathrm{N}^{\prime}$-acetyl-3-indolylmethanamine (Pedras et al., 2011). This detoxification by A. brassicicola is highly detrimental to the host plant because brassinin is not only a crucifer phytoalexin, but also a biosynthetic precursor for several plant defense metabolites. Therefore, detoxification of brassinin by $A$. brassicicola is important for its pathogenicity. In this study, disruption of AbSte 7 reduced brassinin detoxification by $A$. brassicicola. On PDA containing 0.1 or $0.2 \mathrm{M}$ brassinin, the WT strain grew normally, but AbSte 7 disruption mutants M1 and M2 significantly reduced the growth. BHAb, an inducible enzyme produced by $A$. brassicicola, catalyzes the detoxification of brassinin converting to 3-indolylmethanamine (Pedras and Minic, 2012). The expression levels of the $B H A b$ gene in the two $\triangle A b S t e 7$ mutants were severely down-regulated compared with those in WT strain during host-pathogen interaction. These results indicate that $\mathrm{Ab}-$ Fus3-MAPK pathway, together with AbSlt2- and AbHog1MAPK pathways (Joubert et al., 2011), was also involved in phytoalexin detoxification in A. brassicicola. The reduced phytoalexin detoxification capacity may be responsible for the reduced virulence of the $\triangle A b S t e 7$ mutants.

ROS play a major role in pathogen-plant interactions. Microbial infection results in the rapid and transient production of large amounts of ROS in hosts (Apel and Hirt, 2004). Therefore, the pathogen must be able to overcome oxidative stress in order to survive and successfully colonize the host. In the past few years, ROS detoxification has been demonstrated to be vital for the pathogenicity of many fungi (Lin et al., 2009, 2010, 2011; Molina and Kahmann, 2007; Yang and Chung, 2012). In A. alternata, signaling pathways involved in the response to oxidative stress are absolutely required for pathogenicity (Lin et al., 2009, 2010, 2011; Yang and Chung, 2012). There has been limited information regarding the role of ROS detoxification in the pathogenicity of $A$. brassicicola. When $\mathrm{H}_{2} \mathrm{O}_{2}$ detoxification by the $\triangle A b S t e 7$ and WT strains was analyzed, the $\triangle A b S t e 7 \mathrm{M} 1$ and M2 mutants degraded only 32.92 and $28.82 \%$, respectively, of $\mathrm{H}_{2} \mathrm{O}_{2}$ after a 75 -min incubation compared to $58.82 \%$ by WT. The reduced ROS detoxification may be responsible for the defects in pathogenicity displayed by the $\triangle A b S t e 7$ mutants.

CWDEs play important roles in the pathogenesis of necrotrophic fungi (Van Kan, 2006). Generally, these necrotrophic fungi kill host cells directly or by inducing programmed cell death with toxins, and then decompose these cells using CWDEs to facilitate colonization and the release of carbohydrates for consumption (Choquer et al., 2007). The importance of CWDE genes in virulence has been demonstrated in many fungal species, including Botrytis cinerea, Fusarium oxysporum, and Cochliobolus carbonum. In $B$. cinerea, inactivation of xyn11A, a $\beta-1$, 4-xylanase gene, reduced lesion outgrowth by more than $70 \%$ (Brito et al., 2006). However, the role of CWDEs in the pathogenesis of A. brassicicola has not yet been determined. Due to functional redundancy, deletion of an individual gene encoding hydrolase results in little or no change in the pathogenicity of $A$. brassicicola (Cho, 2015). Therefore, we first analyzed carbohydrate utilization and then hydrolytic enzyme activity in the $\triangle A b S t e 7$ mutant. As shown in Fig. 4, AbSte7 negatively regulated the activity of some hydrolytic enzymes during saprophytic growth under nutrient-poor conditions. When the activity of the CWDEs was measured in host tissues infected by the WT and $\triangle A b S t e 7$ mutant strains, the $\triangle A b S t e 7$ mutant was found to have less $\mathrm{Cx}, \beta-\mathrm{G}, \mathrm{PME}$, PGTE, and PMTE activity than the WT during most infection stages, except PG, which had an activity similar to or higher than that of the WT (Fig. 5). Necrotrophic fungi usually produce toxins and CWDEs to kill host cells to acquire nutrients from dead tissues (Divon and Fluhr 2007). Therefore, the reduced CWDE activity may be responsible for the pathogenicity defect in $\triangle A b S t e 7$. The relationship between the reduced CWDE activity and pathogenicity defect in $\triangle A b S t e 7$ needs to be further analyzed in future work.

In summary, disruption of the AbSte7 gene of A. brassicicola reduced the accumulation of toxic metabolites in culture media, detoxification of brassinin and $\mathrm{H}_{2} \mathrm{O}_{2}$, and the enzymatic activity of some CWDEs during host plant infection. Altogether, these data strongly suggest the MAPKK gene AbSte 7 plays a pivotal role in host infection by $A$. brassicicola.

\section{Acknowledgments}

This work was supported by the Foundation of Shandong Province Modern Agricultural Technology System Innovation Team (No. SDAIT-25-07) and the Natural Science Foundation of China (Grant No. 31300130).

\section{References}

Apel, K. and Hirt, H. 2004. Reactive oxygen species: metabolism, oxidative stress, and signal transduction. Annu. Rev. 
Plant Biol. 55:373-399.

Brito, N., Espino, J. J. and González, C. 2006. The endo-beta-1,4xylanase xyn11A is required for virulence in Botrytis cinerea. Mol. Plant-Microbe Interact. 19:25-32.

Chen, L. H., Lin, C. H. and Chung, K. R. 2012. Roles for SKN7 response regulator in stress resistance, conidiation and virulence in the citrus pathogen Alternaria alternata. Fungal Genet. Biol. 49:802-813.

Chen, L. Y., Price, T. V. and Silvapulle, M. J. 2005. Dark leaf spot (alternaria brassicicola) on Chinese cabbage: spatial patterns. Aust. J. Agric. Res. 56:699-714.

Cho, Y. 2015. How the necrotrophic fungus Alternaria brassicicola kills plant cells remains an enigma. Eukaryot. Cell 14:335-344.

Cho, Y., Jang, M., Srivastava, A., Jang, J.-H., Soung, N.-K., Ko, S.-K., Kang, D.-O., Ahn, J. S. and Kim, B. Y. 2015. A pectate lyase-coding gene abundantly expressed during early stages of infection is required for full virulence in Alternaria brassicicola. PLoS One 10:e0127140.

Choquer, M., Fournier, E., Kunz, C., Levis, C., Pradier, J., Simon, A. and Viaud, M. 2007. Botrytis cinerea virulence factors: new insights into a necrotrophic and polyphageous pathogen. FEMS Microbiol. Lett. 277:1-10.

Chung, K. R. 2012. Stress Response and Pathogenicity of the Necrotrophic Fungal Pathogen Alternaria alternata. Scientifica 2012:635431.

Cousin, A., Mehrabi, R., Guilleroux, M., Dufresne, M., Van Der Lee, T., Waalwijk, C., Langin, T. and Kema, G. H. 2006. The MAP kinase-encoding gene MgFus3 of the non-appressorium phytopathogen Mycosphaerella graminicola is required for penetration and in vitro pycnidia formation. Mol. Plant Pathol. 7:269-278.

Divon, H. H. and Fluhr, R. 2007. Nutrition acquisition strategies during fungal infection of plants. FEMS Microbiol. Lett. 266:65-74.

Gustin, M. C., Albertyn, J., Alexander, M. and Davenport, K. 1998. MAP kinase pathways in the yeast Saccharomyces cerevisiae. Microbiol. Mol. Biol. Rev. 62:1264-1300.

Hamel, L. P., Nicole, M. C., Duplessis, S. and Ellis, B. E. 2012. Mitogen-activated protein kinase signaling in plant-interacting fungi: distinct messages from conserved messengers. Plant Cell 24:1327-1351.

Hou, Z., Xue, C., Peng, Y., Katan, T., Kistler, H. C. and Xu, J. R. 2002. A mitogen-activated protein kinase gene (MGV1) in Fusarium graminearum is required for female fertility, heterokaryon formation, and plant infection. Mol Plant-Microbe Interact. 15:1119-1127.

Jenczmionka, N. J., Maier, F. J., Lösch, A. P. and Schäfer, W. C. 2003. Mating, conidiation and pathogenicity of Fusarium graminearum, the main causal agent of the head-blight disease of wheat, are regulated by the MAP kinase gpmk1. Curr. Genet. 43:87-95.

Joubert, A., Bataille-Simoneau, N., Campion, C., Guillemette, T., Hudhomme, P., Iacomi-Vasilescu, B., Leroy, T., Pochon, S.,
Poupard, P. and Simoneau, P. 2011. Cell wall integrity and high osmolarity glycerol pathways are required for adaptation of Alternaria brassicicola to cell wall stress caused by brassicaceous indolic phytoalexins. Cell Microbiol. 13:62-80.

Lev, S., Sharon, A., Hadar, R., Ma, H. and Horwitz, B. A. 1999. A mitogen-activated protein kinase of the corn leaf pathogen Cochliobolus heterostrophus is involved in conidiation, appressorium formation, and pathogenicity: diverse roles for mitogen-activated protein kinase homologs in foliar pathogens. Proc. Natl. Acad. Sci. U.S.A. 96:13542-13547.

Lin, C. H., Yang, S. L. and Chung, K. R. 2009. The YAP1 homolog-mediated oxidative stress tolerance is crucial for pathogenicity of the necrotrophic fungus Alternaria alternata in citrus. Mol. Plant-Microbe Interact. 22:942-952.

Lin, C. H., Yang, S. L., Wang, N. Y. and Chung, K. R. 2010. The FUS3 MAPK signaling pathway of the citrus pathogen Alternaria alternata functions independently or cooperatively with the fungal redox-responsive AP1 regulator for diverse developmental, physiological and pathogenic processes. Fungal Genet. Biol. 47:381-391.

Lin, C. H., Yang, S. L. and Chung, K. R. 2011. Cellular responses required for oxidative stress tolerance, colonization, and lesion formation by the necrotrophic fungus Alternaria alternata in citrus. Curr. Microbiol. 62:807-815.

MacKinnon, S. L., Keifer, P. and Ayer, W. A. 1999. Components from the phytotoxic extract of Alternaria brassicicola, a black spot pathogen of canola. Phytochemistry 51:215-221.

Molina, L. and Kahmann, R. 2007. An Ustilago maydis gene involved in $\mathrm{H}_{2} \mathrm{O}_{2}$ detoxification is required for virulence. Plant Cell 19:2293-2309.

Moriwaki, A., Kihara, J., Mori, C. and Arase, S. 2007. A MAP kinase gene, BMK1, is required for conidiation and pathogenicity in the rice leaf spot pathogen Bipolaris oryzae. Microbiol. Res. 162:108-114.

Nishimura, S. and Kohmoto, K. 1983. Host-specific toxins and chemical structures from alternaria species. Annu. Rev. Phytopathol. 21: 87-116.

Oka, K., Akamatsu, H., Kodama, M., Nakajima, H., Kawada, T. and Otani, H. 2005. Host-specific AB-toxin production by germinating spores of Alternaria brassicicola is induced by a host-derived oligosaccharide. Physiol. Mol. Plant Pathol. 66:12-19.

Otani, H., Kohnobe, A., Kodama, M. and Kohmoto, K. 1998. Involvement of host factors in the production of a protein host-specific toxin by Alternaria brassicicola. In: Molecular Genetics of Host-Specific Toxins in Plant Disease, eds. by K. Kohmoto and O. C. Yoder, pp. 63-69. Proceedings of the 3rd Tottori International Symposium on Host-Specific Toxins, Daisen, Tottori, Japan.

Pearson, G., Robinson, F., Beers Gibson, T., Xu, B., Karandikar, M., Berman, K. and Cobb, M. H. 2001. Mitogen-activated protein (MAP) kinase pathways: regulation and physiological functions. Endocr. Rev. 22:153-183.

Pedras, M. S., Chumala, P. B., Jin, W., Islam, M. S. and Hauck, 
D. W. 2009. The phytopathogenic fungus Alternaria brassicicola: phytotoxin production and phytoalexin elicitation. Phytochemistry 70:394-402.

Pedras, M. S. C. and Yaya, E. E. 2010. Phytoalexins from Brassicaceae: news from the front. Phytochemistry 71:1191-1197.

Pedras, M. S. C., Yaya, E. E. and Glawischnig, E. 2011. The phytoalexins from cultivated and wild crucifers: chemistry and biology. Nat. Prod. Rep. 28:1381-1405.

Pedras, M. S. C. and Minic, Z. 2012. Differential protein expression in response to the phytoalexin brassinin allows the identification of molecular targets in the phytopathogenic fungus Alternaria brassicicola. Mol. Plant Pathol. 13:483-493.

Pedras, M. S. C., Minic, Z. and Hossain, S. 2012. Discovery of inhibitors and substrates of brassinin hydrolase: Probing selectivity with dithiocarbamate bioisosteres. Bioorg. Med. Chem. 20:225-233.

Pedras, M. S. C. and Park, M. R. 2015. Metabolite diversity in the plant pathogen Alternaria brassicicola: factors affecting production of brassicicolin A, depudecin, phomapyrone A and other metabolites. Mycologia 107:1138-1150.

Román, E., Arana, D. M., Nombela, C., Alonso-Monge, R. and Pla, J. 2007. MAP kinase pathways as regulators of fungal virulence. Trends Microbiol. 15:181-190.

Rop, N. K., Kiprop, E. K. and Ochuodho, J. O. 2009. Alternaria species causing black spot disease of Brassicas in Kenya. Afri. Crop Sci. Conf. Proc. 9:635-640.

Ruiz-Roldán, M. C., Maier, F. J. and Schäfer, W. 2001. PTK1, a mitogen-activated-protein kinase gene, is required for conidiation, appressorium formation, and pathogenicity of Pyrenophora teres on barley. Mol. Plant-Microbe Interact. 14:116-125.

Sambrock, J. and Russel, D. 2001. Molecular cloning: A laboratory manual. 3rd ed. Cold Spring Harbor Laboratory
Press, New York, USA. 2344 pp.

Szolar, O. H. 2007. Environmental and pharmaceutical analysis of dithiocarbamates. Anal. Chim. Acta 582:191-200.

Thomma, B. P. 2003. Alternaria spp.: from general saprophyte to specific parasite. Mol. Plant Pathol. 4:225-236.

Van Kan, J. A. 2006. Licensed to kill: the lifestyle of a necrotrophic plant pathogen. Trends Plant Sci. 11:247-253.

Widmann, C., Gibson, S., Jarpe, M. B. and Johnson, G. L. 1999. Mitogen-activated protein kinase: conservation of a threekinase module from yeast to human. Physiol. Rev. 79:143180.

Xu, H., Zhang, Q., Cui, W., Zhang, X., Liu, W., Zhang, L., Islam, M. N., Baek, K.-H. and Wang, Y. 2016. AbSte7, a MAPKK gene of Alternaria brassicicola, is involved in conidiation, salt/oxidative stress, and pathogenicity. J. Microbiol. Biotechnol. 26:1311-1319.

Xu, J. R. 2000. Map kinases in fungal pathogens. Fungal Genet. Biol. 31:137-152.

Xu, J. R. and Hamer, J. E. 1996. MAP kinase and cAMP signaling regulate infection structure formation and pathogenic growth in the rice blast fungus Magnaporthe grisea. Genes Dev. 10:2696-2706.

Yang, S. L. and Chung, K. R. 2012. The NADPH oxidase-mediated production of hydrogen peroxide $\left(\mathrm{H}_{2} \mathrm{O}_{2}\right)$ and resistance to oxidative stress in the necrotrophic pathogen Alternaria alternata of citrus. Mol. Plant Pathol. 13:900-914.

Yang, Z., Bi, Y., Li, Y., Kou, Z., Bao, G., Liu, C., Wang, Y. and Wang, D. 2012. Changes of cell wall degrading enzymes in potato tuber tissue slices infected by Fusarium sulphureum. Sci. Agric. Sinica. 45:127-134 (in Chinese).

Zhao, X., Mehrabi, R. and Xu, J. R. 2007. Mitogen-activated protein kinase pathways and fungal pathogenesis. Eukaryot. Cell 6:1701-1714. 